\title{
RESEARCHES REGARDING THE CIRCULAR INTERPOLATION ALGORITHMS AT CNC LASER CUTTING MACHINES
}

\author{
TÎRNOVEAN Mircea Sorin \\ (Ph.D. Student ), Faculty of Engineering/Department of Industrial Machines and Equipment, "Lucian Blga" \\ University of Sibiu, Sibiu, Romania, tarnovean@yahoo.com
}

\begin{abstract}
This paper presents an integrated simulation approach for studying the circular interpolation regime of CNC laser cutting machines. The circular interpolation algorithm is studied, taking into consideration the numerical character of the system. A simulation diagram, which is able to generate the kinematic inputs for the feed drives of the CNC laser cutting machine is also presented.
\end{abstract}

Key words: CNC laser cutting machine, circular interpolation, motion control, simulation

\section{Introduction}

Laser cutting of sheet metal parts is characterized by high processing speeds, reduced quantity of waste, high accuracies and quality of the processed parts [1, 2]. In comparison with oxy-gas and plasma jet cutting technologies, the laser cutting process leads to a narrower heat-affected zone and a smaller kerf width $[3,4]$.

The motion control system of these machines is a typical 2-axis CNC system, with simultaneous coordinated movements in $\mathrm{XY}$ plane. The $\mathrm{Z}$ axis is also numerically controlled, but simultaneous movements on 3-axis are seldom programmed. The laser cutting machines motion control architecture is quite similar to the control structure of the CNC milling machines and lathes. Typical CNC motion control equipment is based upon rotational or linear servomotors, position and speed transducers and ball screw transmissions. However, there are some important particularities between CNC laser cutting machines and CNC milling machines or lathes. Technological forces (cutting forces and friction forces) are significantly smaller and the feeds are significantly higher within the process of laser cutting.

The motion control system uses advanced servo-control algorithms and techniques (PID control, crosscoupling control, real-time error compensation) in order to minimize the tracking and contouring errors $[5,6,7,8]$.

The motion control strategies are based on dividing the processing paths into linear segments and circular arc and applying accordingly linear and circular interpolation algorithms.

Usually, most of the CNC controllers have a set of control parameters (axes parameters) which may be modified by the user. Of course, the modification of these parameters may significantly affect the machine-tool accuracy.

In order to improve the machine accuracy and its dynamical behavior, a simulation process in needed. An accurate simulation will need, at the input side of the simulation diagram the kinematic inputs generated by the interpolation algorithms. This work will present a method for calculating these kinematic inputs for the circular interpolation algorithms, taking into consideration the numerical character of the CNC controller. A simulation diagram, which is able to show the theoretical toolpath is also presented.

\section{Circular interpolation}

Reference word circular interpolation method involves the issue of reference position inputs on both axes as binary words, at time intervals equal with the CNC equipment sampling time.

For generating a circular trajectory in XOY plane, by means of a circular interpolation algorithm, the main requirement is to held the velocity on the path, $\mathrm{F}$, constant. Consequently, the velocities on $\mathrm{OX}$ and OY axes, $F_{x}$ respectively $F_{y}$ may be expressed as: 
$\left\{\begin{array}{l}F_{x}=F \sin \theta(t) \\ F_{y}=F \cos \theta(t)\end{array}\right.$

where:

$\theta(\mathrm{t})$ - angle at moment $\mathrm{t}$ (fig. 1 ).

The relationship between the angle $\theta(t)$, the path velocity, the angular speed and the circle radius may be expressed by the following equations:

$\theta(t)=\frac{F \cdot t}{R}$

and

$F=\omega \cdot R=\frac{2 \pi}{T} \cdot R$

where:

$\mathrm{R}$ - radius of the circle $[\mathrm{m}]$;

$\mathrm{T}$ - time for generating a whole circle (period) [s].

$\omega-$ angular speed $[\mathrm{rad} / \mathrm{s}]$.

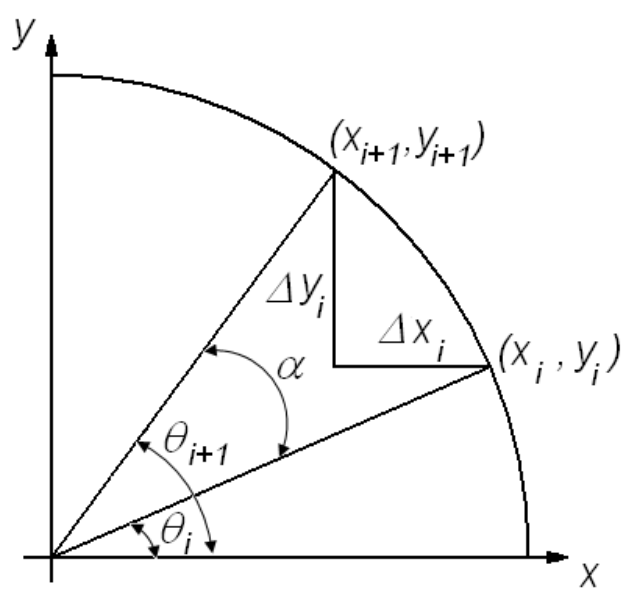

Figure 1: Reference word circular interpolation

Equation (3) leads to:

$\omega=\frac{2 \pi}{T}=\frac{F}{R}$

The circular trajectory is usually approximated by small linear segments. The number of these segments has to be optimized in such way that the deviation from the circular trajectory is smaller than BLU (the basic length unit of the CNC equipment), which is usually $1 \mu \mathrm{m}$.

Figure 1 shows the relationship between two successive interpolated points for the reference word interpolator for a circular arc. Based on figure 1, the following equations may be written:

$\left\{\begin{array}{l}\cos \theta(i+1)=A \cos \theta(i)-B \sin \theta(i) \\ \sin \theta(i+1)=A \sin \theta(i)+B \cos \theta(i)\end{array}\right.$

where: 
$A=\cos \alpha, \quad B=\sin \alpha$

$\theta(i+1)=\theta(i)+\alpha$

and

$\alpha$ - angular displacement increment of the interpolation algorithm (angular step of the interpolation algorithm [degrees].

The coordinates of the final point of the linear segment used for the approximation, $x(i+1), y(i+1)$ may be expressed with the equation:

$\left\{\begin{array}{l}x(i+1)=R \cos \theta(i+1) \\ y(i+1)=R \sin \theta(i+1)\end{array}\right.$

From (5) and (6), relationships which allow the calculation of the current point $i+1$, based upon the coordinates of the previous point i may be written:

$\left\{\begin{array}{l}x(i+1)=A x(i)-B y(i) \\ y(i+1)=A y(i)+B y(i)\end{array}\right.$

The relationship between the linear displacement increments on each axis, $\Delta x(i), \Delta y(i)$ and the coordinates of the points with the linear segment may be expressed as:

$\left\{\begin{array}{l}\Delta x(i)=x(i+1)-x(i)=(A-1) x(i)-B y(i) \\ \Delta y(i)=y(i+1)-y(i)=(A-1) y(i)+B x(i)\end{array}\right.$

The velocities on each axis $F_{x}(i), F_{y}(i)$, may be expressed as:

$$
\left\{\begin{array}{l}
F_{x}(i)=F \frac{\Delta x(i)}{\Delta s(i)} \\
F_{y}(i)=F \frac{\Delta y(i)}{\Delta s(i)}
\end{array}\right.
$$

where:

$\Delta s(i)=\sqrt{(\Delta x(i))^{2}+(\Delta y(i))^{2}}$

By combining (5) and (7), the following equation may be written:

$\left\{\begin{array}{l}x(i+1)=R[A \cos \theta(i)-B \sin \theta(i)] \\ y(i+1)=R[A \sin \theta(i)+B \cos \theta(i)]\end{array}\right.$

Taking into consideration (2) and the numerical character of the interpolation algorithm, the following equation may be written for the time step $T_{i}$ :

$\theta(i)=\frac{F}{R} T_{i}$

The following equation may be also written:

$T_{i}=i \cdot T_{s}$ 
and

$T=n \cdot T_{s}$

where:

$\mathrm{T}_{\mathrm{s}}$ - sampling period [s];

$\mathrm{k}$ - number of samples at the moment Tk;

$\mathrm{n}-$ total number of samples.

Consequently (12) may be rewritten as:

$\left\{\begin{array}{l}x(i+1)=R\left[A \cos \left(2 \pi \cdot \frac{i}{n}\right)-B \sin \left(2 \pi \cdot \frac{i}{n}\right)\right] \\ y(i+1)=R\left[A \sin \left(2 \pi \cdot \frac{i}{n}\right)+B \cos \left(2 \pi \cdot \frac{i}{n}\right)\right]\end{array}\right.$

The total number of samples may be calculated by combining (3) and (15) as:

$n=\frac{2 \pi \cdot R}{F \cdot T_{s}}$

Consequently, (16) may be rewritten as:

$\left\{\begin{array}{l}x(i+1)=R\left[A \cos \left(\frac{F \cdot T_{s}}{R} \cdot i\right)-B \sin \left(\frac{F \cdot T_{s}}{R} \cdot i\right)\right] \\ y(i+1)=R\left[A \sin \left(\frac{F \cdot T_{s}}{R} \cdot i\right)+B \cos \left(\frac{F \cdot T_{s}}{R} \cdot i\right)\right]\end{array}\right.$

The angular displacement increment $\theta_{\text {incr }}$ may be calculated as:

$\theta_{\text {incr }}=\frac{2 \pi}{T} \cdot T_{s}=\frac{F}{R} \cdot T_{s}$

and the linear displacement increment $\mathrm{l}_{\text {incr }}$ as

$$
l_{\text {incr }}=2 R \cdot \frac{\theta_{\text {incr }}}{2 \pi}=\frac{F}{\pi} T_{s}
$$

Equation (20) shows the relationship between the linear displacement increment, velocity on the path and the sampling period.

The minimum angular displacement of the $\mathrm{X}$ and $\mathrm{Y}$ feed drives, $\beta$, may be calculated as:

$\beta=\frac{360^{0}}{N_{\text {imp }}}$

where

$\mathrm{N}_{\mathrm{imp}}$ - number of pulses emitted by the incremental encoder at a full rotation.

The minimum linear displacement of the $\mathrm{X}$ and $\mathrm{Y}$ feed drives, BLU, may be calculated as: 
$B L U=\frac{\beta \cdot p_{s}}{360^{\circ}}$

where

$\mathrm{p}_{\mathrm{s}}$ - step of the leadscrew.

Considering the linear displacement increment $l_{\text {incr }}$, calculated according to (20) being equal with $\mathrm{BLU}$, the following equation may be written:

$B L U=\frac{F}{\pi} T_{s}$

From (23) the sampling period $\mathrm{T}_{\mathrm{s}}$ may be expressed as:

$T_{s}=\frac{B L U \cdot \pi}{F}$

Equation (24) shows the fact that the sampling period $\mathrm{T}_{\mathrm{s}}$ depends on the path velocity, $\mathrm{F}$.

3. Simulation

In order to simulate the interpolation algorithm, a simulation diagram, under Matlab \& Simulink was built (figure 2). The simulation diagram from figure 2 basically uses sample based sine wave blocks. The amplitude of the sine function was made equal to the circle radius, and the overall number of samples n, calculated according to (17) was rounded using Matlab „round” function. The phase shift between sine and cosine signal was introduced by using a certain number of offset samples, which number $\mathrm{N}_{\text {offset }}$, was calculated using the following relation:

$N_{\text {offset }}=-\frac{\frac{\pi}{2} \cdot \operatorname{round}(n)}{2 \pi}$
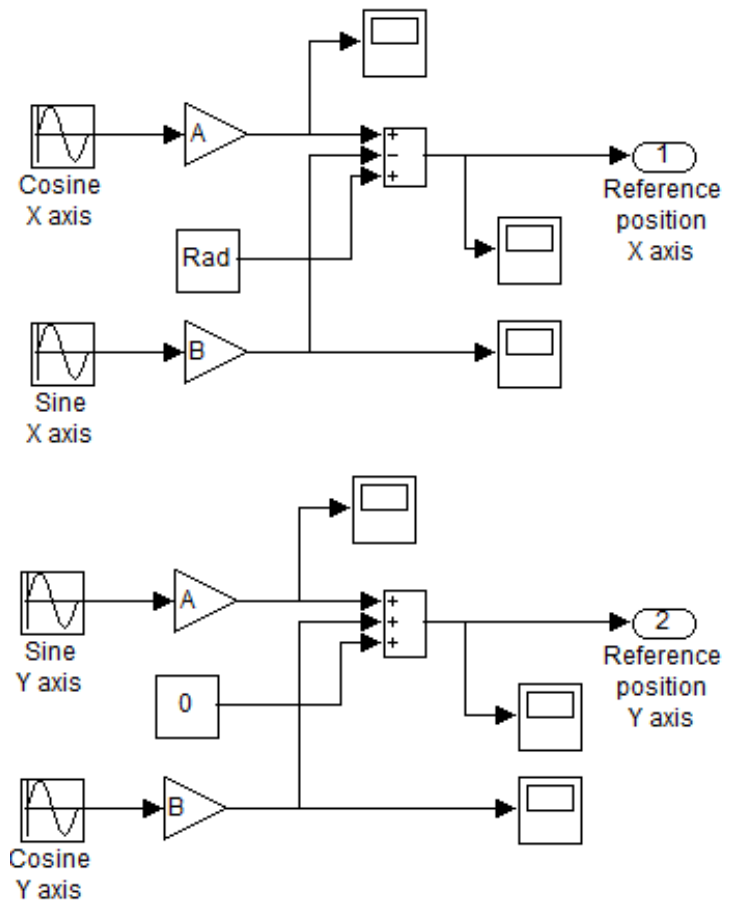

Figure 2: Simulation diagram for the interpolation algorithm

Figure 5 presents a simulated circular toolpath: 


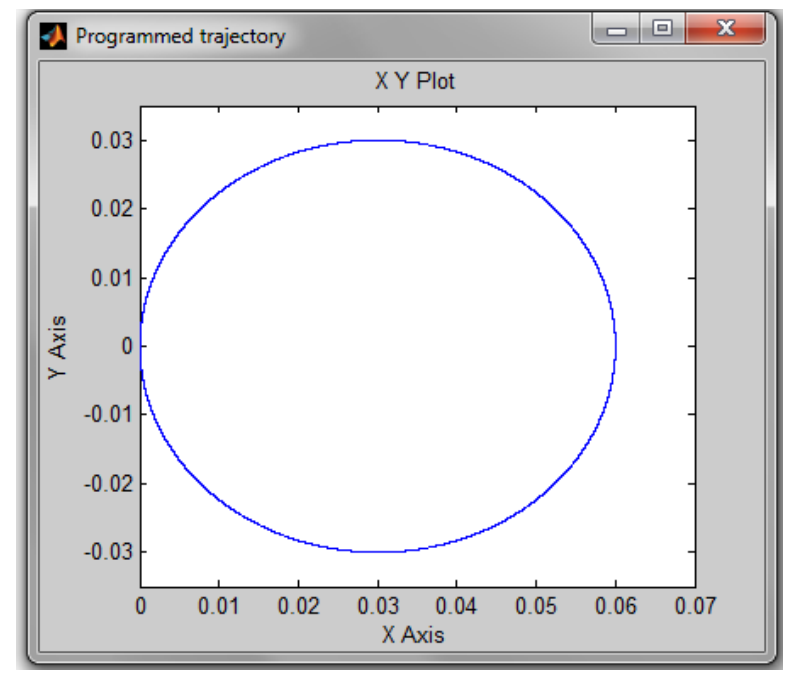

Figure 3: Simulated toolpath

\section{Conclusions}

A diagram for simulating reference word circular interpolation algorithm was presented. The interpolation process is used for generating kinematic inputs for simultaneous movements on two axes.

The algorithm and consequently the simulation diagram were developed in order to generate the input parameters for a more complex simulation process for simulating the behavior of an XY motion control system within a CNC laser cutting machine-tool.

Further researches will be oriented to develop a model of a feed drive using a dc servomotor as actuation device, in order to simulate the behavior of an actual CNC laser cutting machine.

\section{Acknowledgment}

This work was supported by the strategic grant POSDRU/159/1.5/S/133255, Project ID 133255 (2014), co-financed by the European Social Fund within the Sectorial Operational Program Human Resources Development 2007 -2013.

\section{References}

1. J.F. Ready, Industrial applications of lasers, Academy Press, Tokyo, 1997

2. S.D. El Wakil, Processes and design for manufacturing, PWS Publishing Company, Boston, 1998

3. C. Bagger, F.O. Olsen, Pulsed mode laser cutting of sheets for tailored blanks, J. Mater. Process. Technol. 115, pp. 131-135, 2001

4. B.S. Yilbas, Laser cutting quality assessment and thermal efficiency analysis, J. Mater. Process. Technol. 155156, pp. 2106-2115, 2004

5. K. Erkorkmaz, Y. Altintas, High-speed CNC system design, part III: high-speed tracking and contouring control of feed drives, Int. J. Mach. Tools. Manuf. 41, pp. 1637-1658, 2001

6. Y.T. Shin, C.S. Chen, A.C. Lee, A novel cross-coupling control design for Bi-axis motion, Int. J. Mach. Tools. Manuf. 42(14), pp. 1539-1548, 2002

7. J.H. Chin, Y.M. Cheng, J.H. Lin, Improving contour accuracy by Fuzzy-logic enhanced cross-coupled precompensation method, Robot. Comput. Integr. Manuf. 20(1), pp. 65-76, 2004

8. M.Y. Cheng, C.C. Lee, Motion controller design for contour following tasks based on real-time contour error estimation, IEEE Trans. Ind. Electron. 54(3), pp.1686-1695, 2007

9. G. Zhao, Q. Zhang, J. Jiang, Study on Biaxial Linkage for Full-Closed AC Servo System with Modeling and Experiment, Advanced Science Letters, Volume 4, Numbers 6-7, pp. 2009-2014(6), 2011.

10. R.E. Breaz, O. Bologa ,V. Oleksik and G. Racz, Computer Simulation for the Study of CNC Feed Drives Dynamic Behavior and Accuracy, Proceedings of the IEEE Region 8 EUROCON 2007, International Conference on "Computer as a tool”, Warsaw, Poland, pp. 2229-2233, 2007 